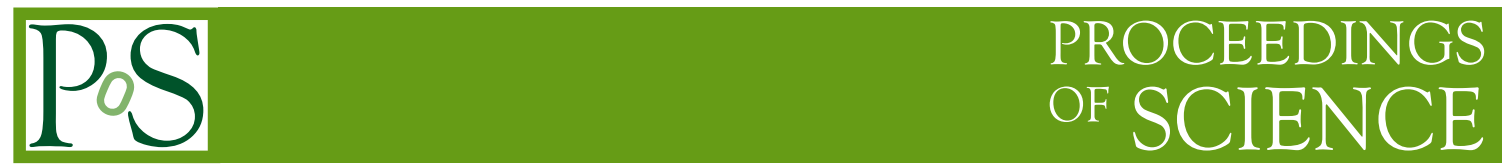

\title{
Looking for new Physics with Pion Decays
}

\author{
Luca Doria*t \\ TRIUMF \\ 4004 Wesbrook Mall, V6T 2A3, Vancouver B.C. (Canada) \\ E-mail: luca@triumf.ca
}

The PIENU experiment at TRIUMF is conceived for measuring the $R=\Gamma\left(\pi^{+} \rightarrow e^{+} v+\pi^{+} \rightarrow\right.$ $\left.e^{+} v \gamma\right) / \Gamma\left(\pi^{+} \rightarrow \mu^{+} v+\pi^{+} \rightarrow \mu^{+} v \gamma\right)$ branching ratio at the $<0.1 \%$ precision level, providing a stringent test of the electron-muon universality hypothesis. The violation of universality can point towards the existence of new physics.

55th International Winter Meeting on Nuclear Physics

23-27 January, 2017

Bormio, Italy

* Speaker.

${ }^{\dagger}$ on behalf of the PIENU Collaboration 


\section{Introduction}

The pion decay branching ratio (including the radiative counterparts)

$$
R=\frac{\Gamma\left(\pi^{+} \rightarrow e^{+} v_{e}+\pi^{+} \rightarrow e^{+} v_{e} \gamma\right)}{\Gamma\left(\pi^{+} \rightarrow \mu^{+} v_{\mu}+\pi^{+} \rightarrow \mu^{+} v_{\mu} \gamma\right)}
$$

is one of the most precisely calculated observables within the Standard Model (SM) [1]. The most recent and precise theoretical calculation [2] gives the following result:

$$
R_{t h}=\frac{g_{e}^{2}}{g_{\mu}^{2}} \frac{m_{e}^{2}}{m_{\mu}^{2}} \frac{\left(m_{\pi}^{2}-m_{e}^{2}\right)^{2}}{\left(m_{\pi}^{2}-m_{\mu}^{2}\right)^{2}}\left[1+\Delta_{e^{2} p^{2}}+\Delta_{e^{2} p^{4}}+\ldots\right]=(1.2352 \pm 0.0001) \times 10^{-4}
$$

The $m_{e}^{2} / m_{\mu}^{2}$ term is a consequence of the helicity suppression mechanism due to the nature of the weak interactions. The ratio $g_{e}^{2} / g_{\mu}^{2}$ is exactly equal to 1 in the case of lepton universality. The first part of the formula containing only the pion and lepton masses is the known tree-level result, while the terms in parentheses represent the radiative and structure-dependent corrections in a chiral perturbation theory expansion.

The experimental values before 2015 come from measurements done at TRIUMF [3] and PSI [4]:

$$
\begin{gathered}
R_{\text {exp }}^{\text {TRIUMF }}=(1.2265 \pm 0.0034(\text { stat }) \pm 0.0044(\text { syst })) \times 10^{-4} \\
R_{\text {exp }}^{P S I}=(1.2346 \pm 0.0035(\text { stat }) \pm 0.0036(\text { syst })) \times 10^{-4}
\end{gathered}
$$

Both experiments are in agreement with the SM. The theoretical precision is over one order of magnitude better than the experimental one, calling for an improved experiment. The goal of the TRIUMF experiment is to improve the precision on $\mathrm{R}$ by a factor of 5, reaching the $0.1 \%$ level. Such a precision will translate in a $0.05 \%$ precision in the test of lepton universality.

The presence of new physics beyond the SM can induce changes in R. Examples of such scenarios include: new pseudoscalar interactions [1], (R-parity violating) SUSY [5], the presence of massive neutrinos [6], excited gauge bosons, leptoquarks [7] and charged Higgs bosons [8]. In particular, at the planned precision level, sensitivity to new pseudoscalar interactions up to a mass scale of $1000 \mathrm{TeV}$ can be achieved.

\section{Experimental Technique and the Detector}

The $75 \mathrm{MeV} / \mathrm{c}$ pion beam is provided by the specially modified M13 secondary beamline at TRIUMF [9]. The primary $500 \mathrm{MeV}, 120 \mu \mathrm{A}$ proton beam from the TRIUMF cyclotron was sent onto a $1 \mathrm{~cm}$ thick beryllium target and the M13 beamline selected particles with an energy loss technique, achieving a final beam composition of $84 \%$ pions, $14 \%$ muons and $2 \%$ positrons. The pions were stopped in an active plastic scintillator target where they decayed in one of the two channels, $\pi^{+} \rightarrow e^{+} v_{e}$ or $\pi^{+} \rightarrow \mu^{+} v_{\mu}$ (see Fig. 1). In the latter case, the muon had $4.1 \mathrm{MeV}$ kinetic energy which is not sufficient for escaping the target and therefore decays at rest via $\mu^{+} \rightarrow e^{+} v_{e} \bar{v}_{\mu}$. Both decays $\pi^{+} \rightarrow e^{+} v_{e}$ and $\pi^{+} \rightarrow \mu^{+} \rightarrow e^{+}$resulted in the emission of a positron from the target. The PIENU detector was designed for measuring time and location of the incoming pion and time, energy and location of the decay positron. In this way, both decays are detected at the 
same time with the same apparatus and acceptance, eliminating many systematic uncertainties in the branching ratio.

The energy and timing characteristics of the two decays are quite different. The positron from the $\pi^{+} \rightarrow \mu^{+} \rightarrow e^{+}$decay chain has a broad energy distribution (0.5-52 MeV) resulting from the three-body $\mu^{+} \rightarrow e^{+} v_{e} \bar{v}_{\mu}$ decay of the muon and the decay time is dominated by the muon decay time $\tau_{\mu}=2.2 \mu \mathrm{s}$. The positron from the $\pi^{+} \rightarrow e^{+} v_{e}$ decay instead has a fixed energy of about 69.8 $\mathrm{MeV}$ and a timing driven by the pion lifetime $\tau_{\pi}=26 \mathrm{~ns}$. Measuring accurately energy and time of the positrons is the key to disentangle the two decay channels. Energy and time distributions are showed in Fig. 2.

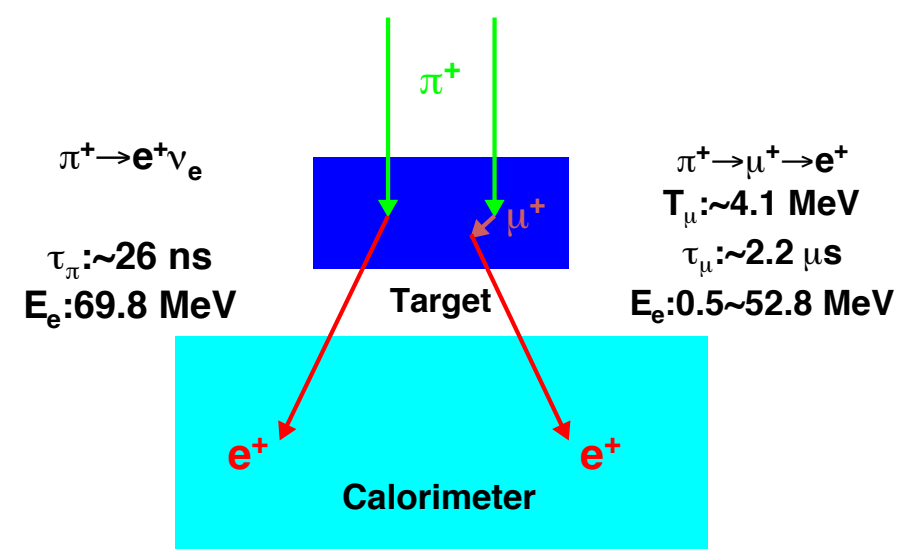

Figure 1: Stopped pion technique used in the PIENU experiment. The muon is fully contained in the target while decay positrons from both decay channels are detected.
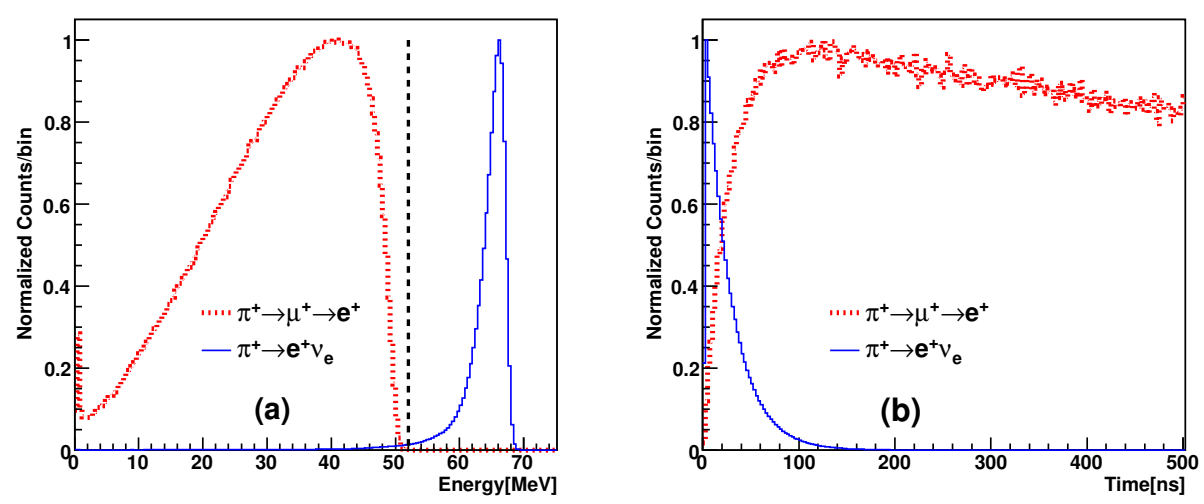

Figure 2: Simulated energy (left) and time (right) distributions for the $\pi^{+} \rightarrow e^{+} v_{e}$ (blue) and $\pi^{+} \rightarrow \mu^{+} \rightarrow e^{+}$ (red) decays. The distributions are normalized for comparison purposes.

The PIENU detector schematic is showed in Fig. 3 and it is described in detail in [10]. The pion beam tracking was provided by two multiwire proportional chambers (WC1/2), each with three planes of wires oriented at $60^{\circ}$ from each other. After WC1/2, the beam was degraded by two plastic scintillators (B1, B2) providing the pion arrival trigger signal and energy loss measurement for 
particle identification and pileup rejection. Pions were stopped in an $8 \mathrm{~mm}$ thick plastic scintillator (B3) where they decayed at rest. Silicon microstrip detectors (S1, S2) were installed before the target for tracking pions and to detect decays in flight. After the target, a third silicon microstrip detector (S3) and a wire chamber (WC3) provided positron tracking. After S3 and WC3 there were two plastic scintillators (T1, T2) for positron detection which provided the decay trigger signal. WC3 and T2 were mounted right in front of a $\mathrm{NaI}(\mathrm{Tl})$ calorimeter crystal $(48 \mathrm{~cm} \times 48 \mathrm{~cm})$ which provided the positron energy measurement. The main calorimeter was surrounded by 97 CsI crystals arranged in a two-layer concentric structure for shower leakage detection. Additional veto scintillators were placed on the beamline, calorimeter and WC3 flanges for covering dead materials. The position of the target with respect to the calorimeter resulted in $20 \%$ acceptance for positrons tracks.

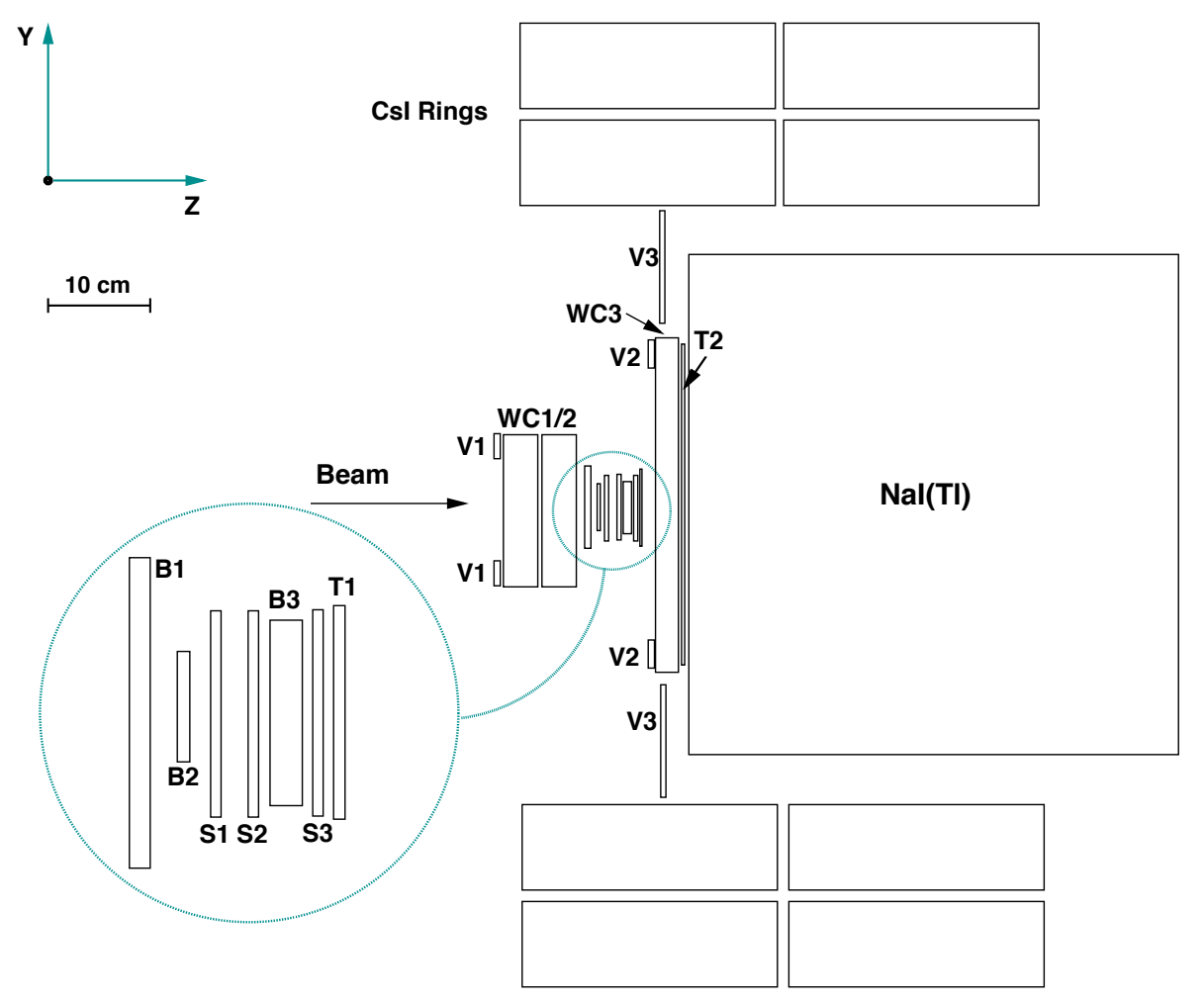

Figure 3: Elevation view of the PIENU detector. The target (B3) region is comprised of wire chambers (WC1/2) and silicon detectors (S1, S2) for pion tracking, plastic scintillators (B1, B2) for pion energy loss measurement, silicon detector (S3) and wire chamber (WC3) for decay positron tracking, and scintillators (T1, T2) for positron time measurement. The calorimeter is a NaI(Tl) crystal surrounded by 97 CsI crystals for shower leakage containment.

All the scintillators were digitized by $500 \mathrm{MHz}$ waveform digitizers. The CsI calorimeter and the silicon detectors were digitized at $60 \mathrm{MHz}$ while for the slower $\mathrm{NaI}(\mathrm{Tl})$ calorimeter a lower rate of $30 \mathrm{MHz}$ was used. The trigger logic was designed for increasing the proportion of $\pi^{+} \rightarrow e^{+} v_{e}$ decays in the data and for accomodating the presence of calibration procedures. The main physics trigger condition was based on a positively identified incoming pion at $t=0 \mathrm{~ns}$ and the presence 
of a decay positron in the $[-300,540] \mathrm{ns}$ time window. The timing window before the pion arrival was inspected for background estimation purposes. The $\pi^{+} \rightarrow \mu^{+} \rightarrow e^{+}$decay was recorded with a prescale factor of 16 , while the $\pi^{+} \rightarrow e^{+} v_{e}$ was enhanced by two trigger conditions based on a decay time shorter than $35 \mathrm{~ns}$ and energy $(\mathrm{NaI}(\mathrm{Tl})+\mathrm{CsI})$ larger than $45 \mathrm{MeV}$. The typical pion stopping rate was $50 \mathrm{kHz}$, while the trigger rate was $600 \mathrm{~Hz}$.

\section{Data Analysis}

The PIENU experiment collected physics data between 2009 and 2012 and here we present the analysis of the 2010 dataset already published in [12]. This corresponds to about $1 / 20$ of the total statistics collected, sufficient to supersede previous experimental results in terms of precision.

A blind analysis procedure was followed and the extracted branching ratio was modified in the analysis software by an unknown factor (within $\pm 1 \%$ ) which was removed only after all the systematic checks and agreement within the collaboration.

The data analysis was based on the timing information provided by the $\mathrm{B} 1$ and $\mathrm{T} 1$ plastic scintillators. The waveforms from the B1 and T1 scintillators were fitted event by event for timing extraction. The energy information was provided by the total energy detected by the calorimeters $(\mathrm{NaI}(\mathrm{Tl})+\mathrm{CsI})$. The final energy spectrum is showed in Fig. 4.

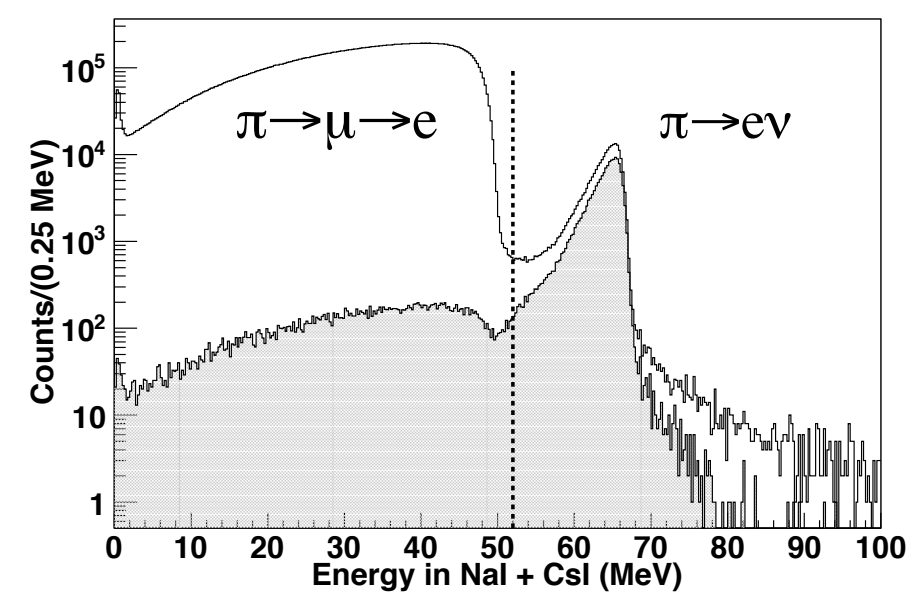

Figure 4: Total energy measured in the calorimeters. The contributions of the two decays are clearly visible. The shaded spectrum is obtained applying suppression cuts in order to reduce $\pi^{+} \rightarrow \mu^{+} \rightarrow e^{+}$events (see Sec. 4). The vertical dashed line shows the cutoff energy $E_{c u t}=52 \mathrm{MeV}$ between the two decays employed in the data analysis.

The data analysis is based on dividing the two decay channels with a cutoff $E_{\text {cut }}=52 \mathrm{MeV}$ in the energy spectrum (the dashed line in Fig. 4). The branching ratio is extracted fitting at the same time the two timing distributions corresponding to the two energy regions (See Fig. 5).

The timing spectrum corresponding to low-energy events $(E<52 \mathrm{MeV})$ was fitted with the following shapes: 


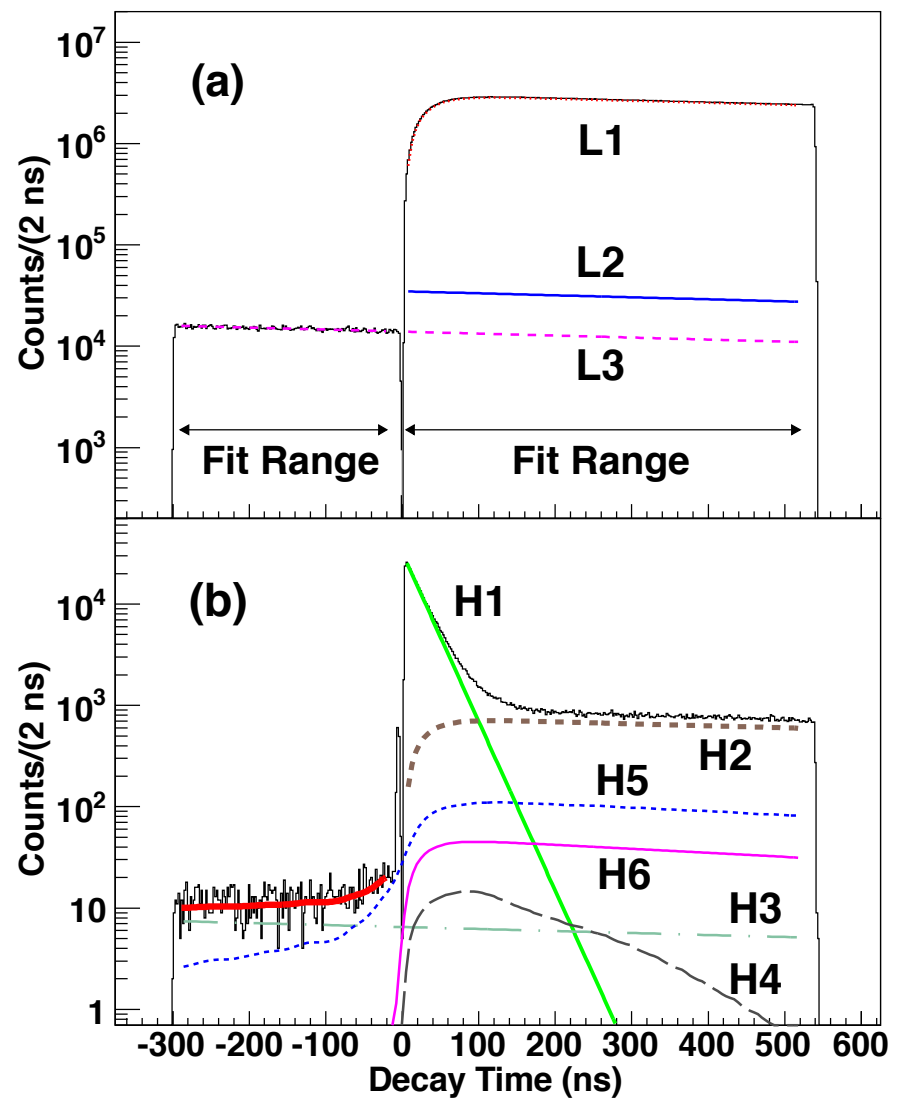

Figure 5: Timing distributions corresponding to the two energy regions $E<52 \mathrm{MeV}$ (top) and $E>52 \mathrm{MeV}$ (bottom). The fitted shapes are reported with different codes and are fully described in the text.

$\mathrm{L} 1(\mathrm{t})=\frac{\lambda_{\pi} \lambda_{\mu}}{\lambda_{\pi}-\lambda_{\mu}}\left(e^{-\lambda_{\mu} t}-e^{-\lambda_{\pi} t}\right)$ for $t>0$ and $\lambda_{\pi / \mu}=1 / \tau_{\pi / \mu}$,

$\mathrm{L} 2(\mathrm{t})=\lambda_{\mu} e^{-\lambda_{\mu} t}$ for $t>0$,

$\mathrm{L} 3(\mathrm{t})=\lambda_{\mu} e^{-\lambda_{\mu} t}$ for any $\mathrm{t}$.

L1 describes the $\pi^{+} \rightarrow \mu^{+} \rightarrow e^{+}$decay chain, L2 the case where the pion decays in flight and L3 accounts for the decay of old muons already present in the target or surrounding materials.

The timing spectrum corresponding to the high-energy events $(E>52 \mathrm{MeV})$ was fitted with the following shapes:

$\mathrm{H} 1(\mathrm{t})=\lambda_{\pi} e^{-\lambda_{\pi} t}$ for $\mathrm{t}>0$,

$\mathrm{H} 2(\mathrm{t})=\frac{\lambda_{\pi} \lambda_{\mu}}{\lambda_{\pi}-\lambda_{\mu}}\left(e^{-\lambda_{\mu} t}-e^{-\lambda_{\pi} t}\right)$ for $\mathrm{t}>0$,

$\mathrm{H} 3(\mathrm{t})=\lambda_{\mu} e^{-\lambda_{\mu} t}$ for any $\mathrm{t}$.

$\mathrm{H} 1$ describes the $\pi^{+} \rightarrow e^{+} v_{e}$ decay, while $\mathrm{H} 2$ and $\mathrm{H} 3$ are analogous to $\mathrm{L} 2$ and $\mathrm{L} 3$ and are present in the high-energy region because of detector resolution effects, pileup and muon radiative decays. The contribution from radiative pion decays is calculated via a Monte Carlo simulation, resulting 
in the shape H4. The shape H5 accounts for two decays happening close enough in time to be integrated by the calorimeter. Also this shape was estimated with a dedicated Monte Carlo which includes the pulse shape and integration time of the $\mathrm{NaI}(\mathrm{Tl})$ and $\mathrm{CsI}$ calorimeters. H6 results from the finite double pulse time resolution of the T1 scintillator and it is estimated from the data by artificially inflating the $\mathrm{T} 1$ resolution and then extrapolating the result to the measured one. The free parameters in the fit were the amplitudes of the L1,2,3 and H1,2,3,5 shapes and the branching ratio $R_{\text {raw }}$ is the ratio of the $\mathrm{H} 1$ and $\mathrm{L} 1$ amplitudes. $\mathrm{H} 4$ was fixed by the known radiative pion branching ratio.

The fitted raw branching ratio was

$$
R_{\text {raw }}=(1.1972 \pm 0.0022(\text { stat }) \pm 0.0005(\text { syst })) \times 10^{-4}
$$

with $\chi^{2} /$ dof $=1.02($ dof $=673)$.

\section{Corrections and Systematic Checks}

\subsection{Tail Correction}

After the extraction of $R_{\text {raw }}$ from the simultaneous fit of the two timing spectra, corrections were needed. The largest correction came from the low-energy tail of the $\pi^{+} \rightarrow e^{+} v_{e}$ decay which extends underneath the overwhelming $\pi^{+} \rightarrow \mu^{+} \rightarrow e^{+}$background prohibiting a direct access to its magnitude. Bacause of the $\pi^{+} \rightarrow e^{+} v_{e}$ events in the tail, the branching ratio resulted too low and a correction was needed.

For estimating the amount of low energy tail, two different strategies were devised. The first one is based on removing the detectors before the calorimeter and injecting a positron beam in it in order to obtain its detailed response (see Fig. 6). Rotating the detector with respect to the beam axis, it was possible to investigate the response in energy and angle. In the calorimeter response, additional peaks were detected and detailed Monte Carlo simulation showed that they arise from photonuclear reactions on Iodine and subsequent emission of neutrons which leave the detector [11]. The low energy tail derived from the positron beam data was regarded as an upper limit to the true value, since the beam itself might induce a slightly larger tail due to scattering effects in the beamline. The upper limit to the low energy tail fraction below $52 \mathrm{MeV}$ was found to be $3.19 \pm 0.03$ (stat) \pm 0.08 (syst) $\%$.

The second strategy resulted instead in a lower bound to the low energy tail. For estimating it, $\pi^{+} \rightarrow \mu^{+} \rightarrow e^{+}$events were suppressed using an early decay-time region 5-35 ns, pulse shape and total pion energy deposited in B1, B2, S1, S2, B3, and measurements of the straightness of the pion track (see [13] for a detailed explanation). The resulting suppressed spectrum is the shaded histogram in Fig. 4. The remaining backgrounds were subtracted using the fact that the backgroundsuppressed spectrum in a low-energy region contained a negligible $\pi^{+} \rightarrow e^{+} v_{e}$ tail contribution. The area of the low-energy region was scaled to the full region (< Ecut) using the known background distributions. Since the total pion energy cut tended to remove $\pi^{+} \rightarrow e^{+} v_{e}$ events with Bhabha scattering (resulting in a larger energy deposit in B3), a Monte Carlo correction to the extracted low energy tail was needed. The final lower limit was $2.95 \pm 0.07$ (stat) \pm 0.08 (syst)\%.

Combining the upper and lower bounds to the low energy tail resulted in a multiplicative correction to the branching ratio of $1.0316 \pm 0.0012$. 

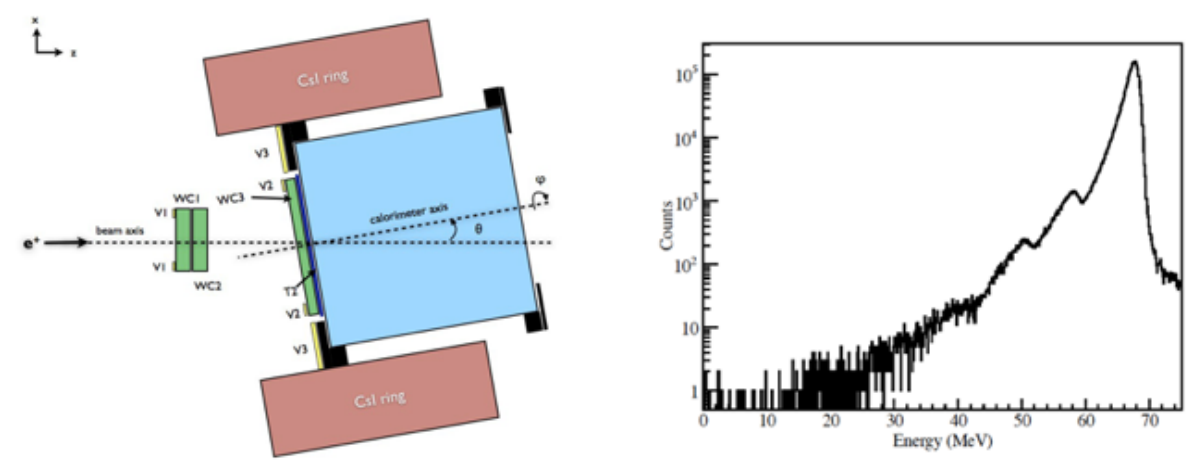

Figure 6: (Left) Schematic of the calorimeter's lineshape measurement. Only WC1/2, T2, WC3, the NaI(Tl) and CsI calorimeters were used. The positron beam was injected at different angles and energies for characterizing the detector's response. (Right) The lineshape in the case of an injected beam at $0^{\circ}$ is showed. The peaks due to photonuclear reactions and neutron escape are evident.

\subsection{Muon Decays in Flight Correction}

The case in which the pion decays at rest and the muon decays in flight in the target ( $\mu \mathrm{DIF})$ must be treated as a correction to the branching ratio, since in this case the timing of such an event is not distinguishable to a $\pi^{+} \rightarrow e^{+} v_{e}$ decay. The amount of $\mu \mathrm{DIF}$ with $E>E_{\text {cut }}$ was estimated with a Monte Carlo simulation and it was found to be $0.2 \%$ of the $\pi^{+} \rightarrow \mu^{+} \rightarrow e^{+}$events. The $\mu$ DIF distribution compared to the decay at rest one is showed in Fig. 7.

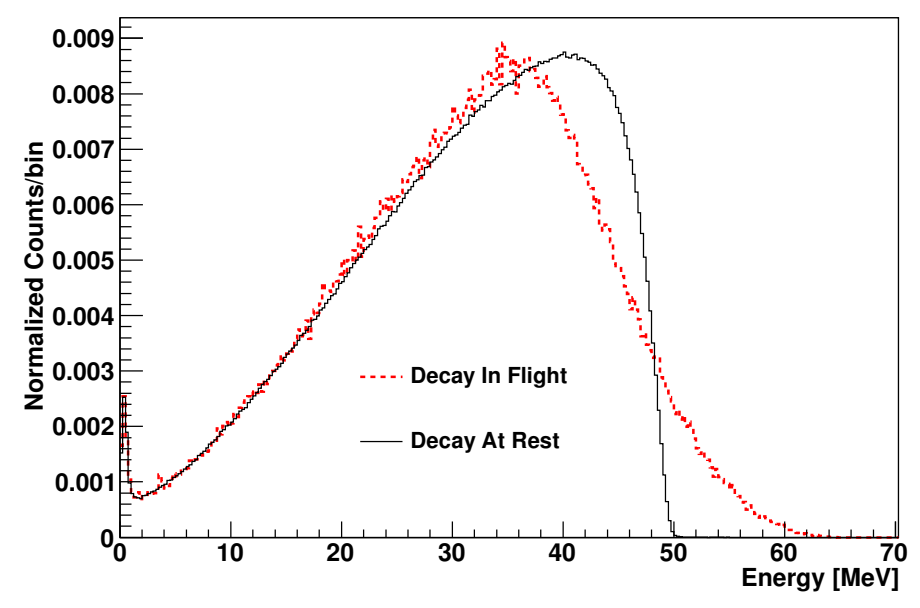

Figure 7: Simulated distributions of $\pi^{+} \rightarrow \mu^{+} \rightarrow e^{+}$events in the case where the pion decays at rest and the muon decays either at rest (black) or in flight (red) in the target B3.

\subsection{Timing Correction}

Possible energy-dependent effects on the pion arrival time were studied using positrons in the beam at momenta $10-70 \mathrm{MeV} / \mathrm{c}$, and with positrons from muons stopped at the center of B3 by lowering the beam momentum to $62 \mathrm{MeV} / \mathrm{c}$. The multiplicative correction from this effect was $1.0004 \pm 0.0005$. 


\subsection{Systematic Checks}

The stability of $R_{\text {raw }}$ was tested for dependence on many parameters: fitting range, histogram binning, pile-up cuts, acceptance cut and $E_{\text {cut }}$. In particular, since the tail correction changes with acceptance and $E_{c u t}$, finding a stable branching ratio as a function of these two cuts gives confidence in the knowledge of the low energy tail shape. The stability of $R_{\text {raw }}$ against the acceptance (selected radius with WC3) and $E_{\text {cut }}$ are showed in Fig. 8.
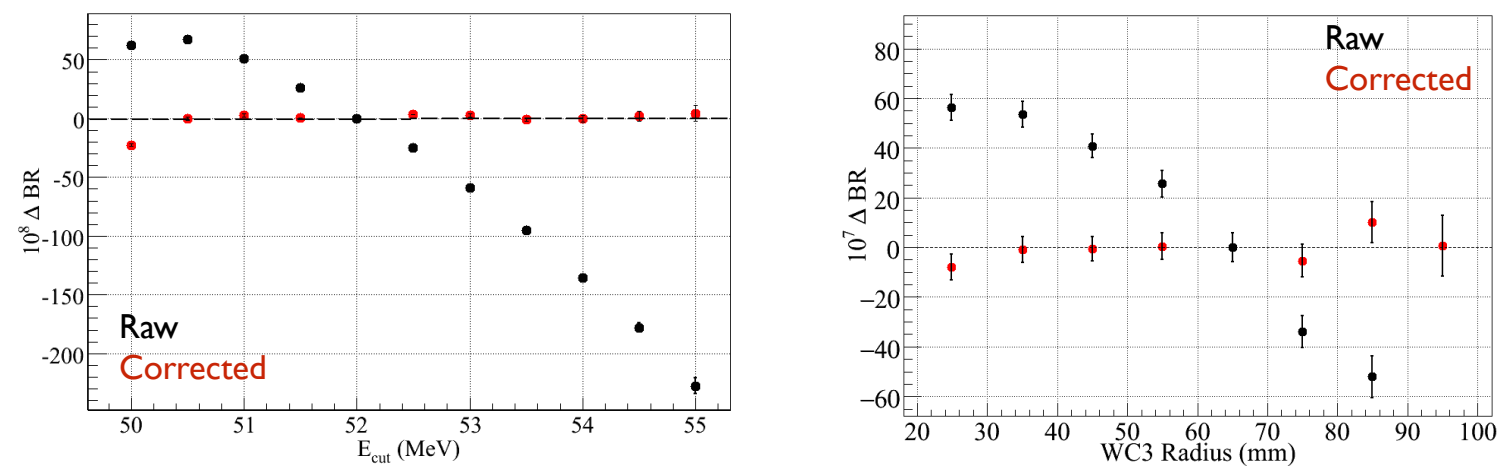

Figure 8: Branching ratio as a function of $E_{\text {cut }}$ (left) and the acceptance radius in WC3 (right). The black points are the uncorrected $R_{\text {raw }}$ while the red ones are corrected for the low energy tail and $\mu$ DIF.

\section{Results and Conclusion}

The final branching ratio after all the corrections and the removal of the blinding factor was found to be

$$
R=(1.2344 \pm 0.0023 \text { (stat) } \pm 0.0019 \text { (syst) }) \times 10^{-4}
$$

consistent with previous experimental results and with the SM prediction. This result improves the test of lepton universality for the first two SM generations by a factor of two:

$$
\frac{g_{e}}{g_{\mu}}=0.9996 \pm 0.0012
$$

These results represent $1 / 20$ of the total data collected and the full analysis is undergoing. General improvements to the analysis strategy and to the simulation will lead to lower systematic uncertainties and the final goal of the experiment is to obtain $0.1 \%$ precision on the branching ratio. The collected data will also be used to improve limits on massive neutrinos in $\pi^{+} \rightarrow e^{+} v_{e}$ decays [13] as well as to search for other beyond SM particles, like e.g. Majorons [14].

\section{References}

[1] Rare Pion and Kaon Decays, D. Bryman, W. Marciano, R. Tschirhart, T. Yamanaka, Ann. Rev. Nucl. Part. Sci. 61, 331 (2011).

[2] V. Cirigliano and I. Rosell, JHEP 0710, 005 (2007).

[3] D.I. Britton et al., Phys. Rev. Lett. 68, 3000 (1992). and D.I. Britton et al., Phys. Rev. D49, 28 (1994). 
[4] G. Czapek et al., Phys. Rev. Lett. 70, 17 (1993).

[5] M.J. Ramsey-Musolf, S. Su and S. Tulin, Phys. Rev. D76, 095017 (2007).

[6] H. Lacker and A. Menzel, JHEP 07, 006 (2010).

[7] S.Davidson, D. Bailey and B. Campbell, Z. Phys. C61, 613 (1994).

[8] D. Bryman et al., Ann. Rev. Nucl. Part. Sci. 61, 331 (2011).

[9] A. Aguilar-Arevalo et al., Nucl. Instrum. Meth. A609, 102 (2009).

[10] A. Aguilar-Arevalo et al., Nucl. Instrum. Meth., A791, 38-46 (2015).

[11] A. Aguilar-Arevalo, et al., Nucl. Instrum. Meth. A621, 188191 (2010).

[12] A. Aguilar-Arevalo, et al., Phys. Rev. Lett. 115, 071801 (2015).

[13] M. Aoki et al., Phys. Rev. D84, 052002 (2011).

[14] C.E. Picciotto, et al., Phys. Rev. D37, 5, 1131-1133 (1988). 